\title{
Damage Detection in CFRP Plates Using Spectral Entropy
}

\author{
E. Castro, ${ }^{1}$ P. Moreno-García, ${ }^{2}$ and A. Gallego ${ }^{3}$ \\ ${ }^{1}$ Applied Physics Department, Universidad Politécnica de Cartagena, 30202 Cartagena, Murcia, Spain \\ ${ }^{2}$ INEGI, Instituto de Engenharia Mecânica e Gestão Industrial, Campus da FEUP, Rua Dr. Roberto Frias 400, \\ 4200-465 Porto, Portugal \\ ${ }^{3}$ Applied Physics Department, Universidad de Granada, 18071 Granada, Spain
}

Correspondence should be addressed to E. Castro; enrique.castro@upct.es

Received 7 July 2013; Accepted 5 February 2014; Published 20 May 2014

Academic Editor: Nuno Maia

Copyright (C) 2014 E. Castro et al. This is an open access article distributed under the Creative Commons Attribution License, which permits unrestricted use, distribution, and reproduction in any medium, provided the original work is properly cited.

Damage detection techniques using vibrations are based on measuring the changes in the vibration parameters of a structure. This paper studies the viability of the spectral entropy as a new damage detection parameter to detect the presence of damage in a composite fiber reinforced polymers (CFRP) plate. To carry out this study, the vibrations in a CFRP plate with and without damage were measured and the correlation between damage and spectral entropy has been researched.

\section{Introduction}

Structural health monitoring (SHM) is very important in aerospace industry due to the high responsibility of the aeronautical structures and their high maintenance costs. So, the developing of reliable damage detection techniques with aerospace application is a very important issue. As composite structures are becoming very important in aerospace engineering, because their high stiffness and less weight, it is very interesting the investigation of damage detection methods for them.

This paper investigates the use of a new damage detection technique in a composite plate. The damage detection technique is based on the use of the entropy of the frequency response function (FRF), which is called spectral entropy (SE), as damage index. The FRF of a structure depends of its geometry and materials, and it is well known that a fracture or a crack produces a change in the FRF [1], which can be used to identify the damage. But in the FRF there is a lot of information, and it is necessary to analyse all this information to detect the damage. Usually the information is reduced in some way to have only one parameter which can be used as damage index. The SE is another way to do it. It is the entropy of the FRF, so it is a single parameter that characterizes the amplitude distribution of the FRF. The principal advantage over other damage indexes based on changes in the natural frequencies is that the SE depends on the distribution of amplitude of the FRF. So, a shift or a change in the global amplitude of the FRF due to external factors does not affect the value of the SE, making it very robust against a change in ambient conditions.

To evaluate the performance of the SE as a damage index, an experiment has been carried out. The vibrations of a CFRP plate with and without damage have been measured, and the FRF and SE have been obtained. As a final result, a correlation between the damage and SE has been found.

\section{Experimental Description}

The properties of the CFRP plate used in the experiment are listed in Table 1 . The plate was excited with a random vibration of frequencies up to $1500 \mathrm{~Hz}$ by means of a piezoelectric actuator. The vibrations of the plate were measured with four piezoelectric sensors located symmetrically around the center of the plate. The actuator was located at the center of the plate. The positions and properties of the sensors and actuators are shown in Table 2. The four sides of the plate were clamped to a rigid frame on an antivibration table. Piezoelectric sensors were connected with STP cables to the Brüel and Kjær PULSE input channels in order to cancel out electromagnetic interference (EMI) and crosstalk 


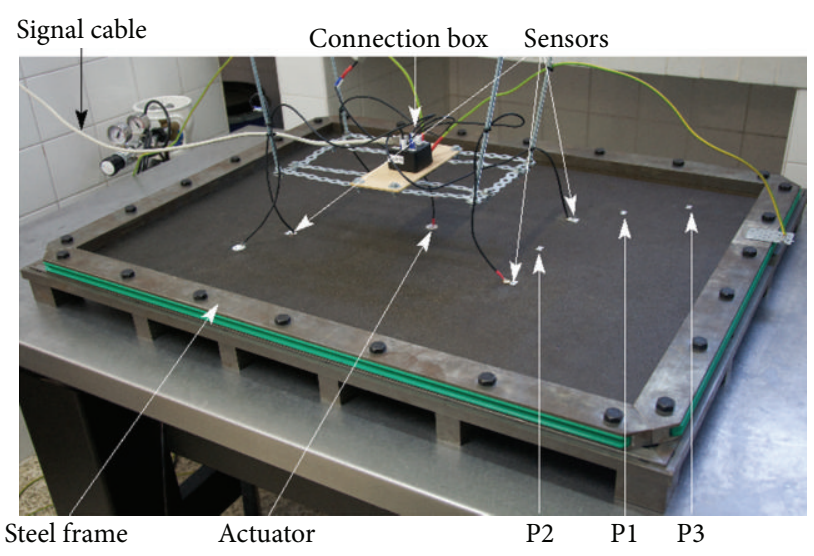

FIgURE 1: Plate under study and experimental arrangement.

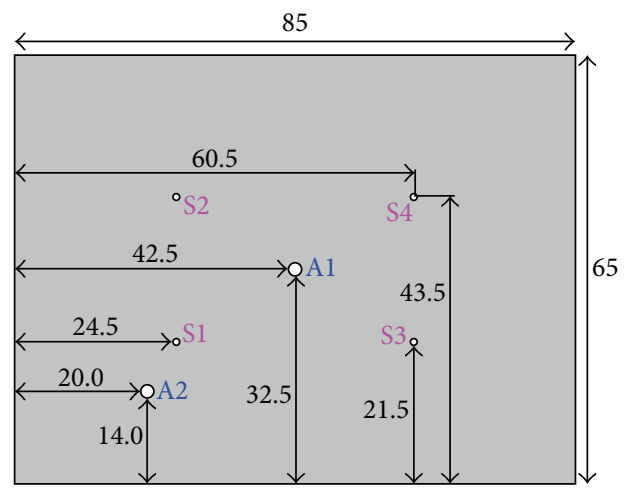

FIgURE 2: Dimensions of the CFRP plate and positions of the damage (P1, P2, and P3) and the actuator (A1).

TABLE 1: Properties of the CFRP plate.

\begin{tabular}{lc}
\hline Side (a) length & $85 \mathrm{~cm}$ \\
Side (b) length & $65 \mathrm{~cm}$ \\
Longitudinal Young's moduli & $1.50 \times 10^{11} \mathrm{~Pa}$ \\
Transversal Young's moduli & $1.15 \times 10^{10} \mathrm{~Pa}$ \\
Poisson's ration & 0.42 \\
Shear moduli & $5.0 \times 10^{9} \mathrm{~Pa}$ \\
Density & $1560 \mathrm{~kg} / \mathrm{m}^{3}$ \\
Mass & $4.75 \mathrm{~kg}$ \\
Layer thickness & $0.188 \mathrm{~mm}$ \\
Number of layers & 12 \\
Stacking sequence & {$[0 / 90]_{3 \mathrm{~S}}$} \\
\hline
\end{tabular}

between neighbouring pairs and external sources. The input signal was also generated with the PULSE output port. The piezosystem EPA-104 amplifier boosted the PULSE output signal to the actuator. To minimize the EMI and electrical noise, all equipment, connection boxes, cable shields, and the frame were connected to an independent ground system. To reduce the influence of external vibrations, the frame was fixed to an antivibration table and the measurements were carried out at night with minimal ambient noise. Figure 1 shows the plate with the attached sensors, actuator, and damage locations.

The damage was simulated by masses attached to the plate with weights, $6.7 \mathrm{~g}$ (M1), $12.8 \mathrm{~g}$ (M2), $24.7 \mathrm{~g}$ (M3), $39.6 \mathrm{~g}$ (M4), $85.8 \mathrm{~g}$ (M5), and $192.9 \mathrm{~g}$ (M6), at three different positions shown in Table 3. In Figure 2 can be seen a diagram of the different positions of the damage in the plate. The plate was placed in a horizontal position and the mass was put on it without any fixing mechanism. The amplitude of the vibrations was low, so the mass stayed in its position while the experiment was performing. As the mass was free, it is possible that it can "jump" on the plate when it goes down. During the experiment no evidence of this jump was noticed.

This kind of damage has the advantages of being very well controlled and nondestructive, which is especially important to validate damage detection and evaluation methods. Furthermore, the addition of masses produces a rise of the local density, whose effect in the vibrational behaviour of the plate is similar to a reduction of the stiffness, as the vibrational frequencies depends on $\sqrt{k / \rho}$, where $k$ is the stiffness and is the density. More details of the experiment can be found in $[2,3]$.

\section{Spectral Entropy}

Entropy is a thermodynamic magnitude that can be used to calculate the quantity of energy that cannot be transformed into work. From a microscopic point of view, it is a measure of the disorder of a system. Entropy is used too in information theory, where it is related with the uncertainty in a random variable, where it is called Shannon entropy. For our purposes, we are going to use the interpretation of entropy as a measure of the disorder of a system but applying it to the frequency distribution of a signal. The Shannon entropy can be calculated with the equation:

$$
E=-\sum_{i=1}^{N} p_{i} \log \left(p_{i}\right)
$$

where $p_{i}$ is the probability of the system of being in the state $i$ and $N$ is the total number of states.

In order to use (1) to calculate the SE, the FRF is normalized and then each frequency peak is considered a state $i$ and its amplitude is assumed to be its probability $p_{i}$. So, the SE is calculated using the expression:

$$
\mathrm{SE}=-\sum_{i=1}^{N} \frac{A\left(f_{i}\right)}{A_{T}} \log \left(\frac{A\left(f_{i}\right)}{A_{T}}\right)
$$

where $A\left(f_{i}\right)$ is the amplitude of the frequency peak $f_{i}$ and $A_{T}=\sum_{i=1}^{N} A\left(f_{i}\right)$. Only the peaks in the FRF are taken into account in the calculation of the SE.

The SE measures the disorder of the amplitude distribution. If all the energy is concentrate in few frequencies, the FRF is very ordered and the SE has a low value. In the same 

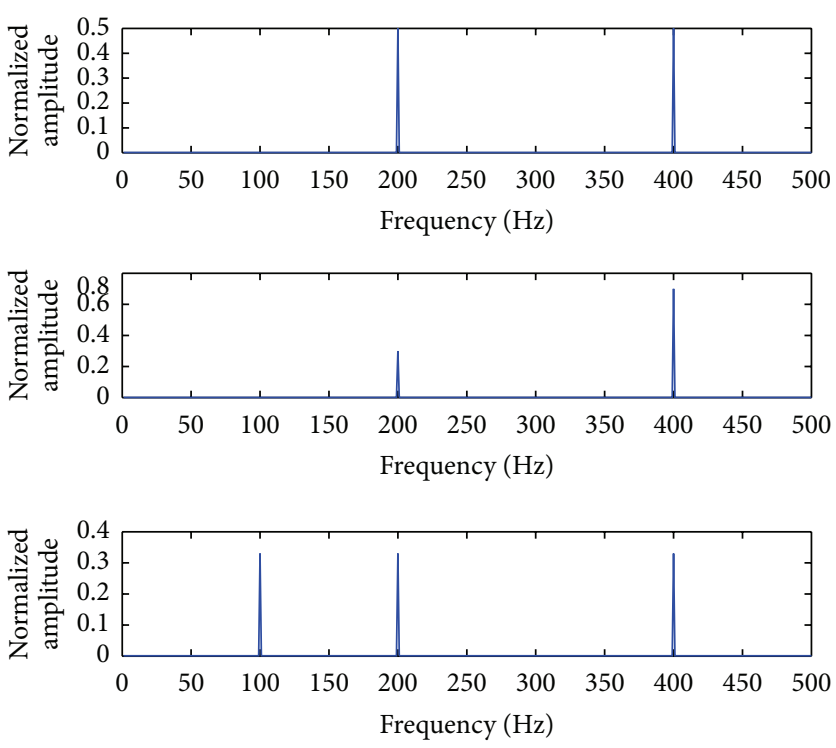

FIGURE 3: FRFs used as examples of the dependency of SE with the amplitude distribution. Their values of SE are 3.163, 3.108, and 3.443.
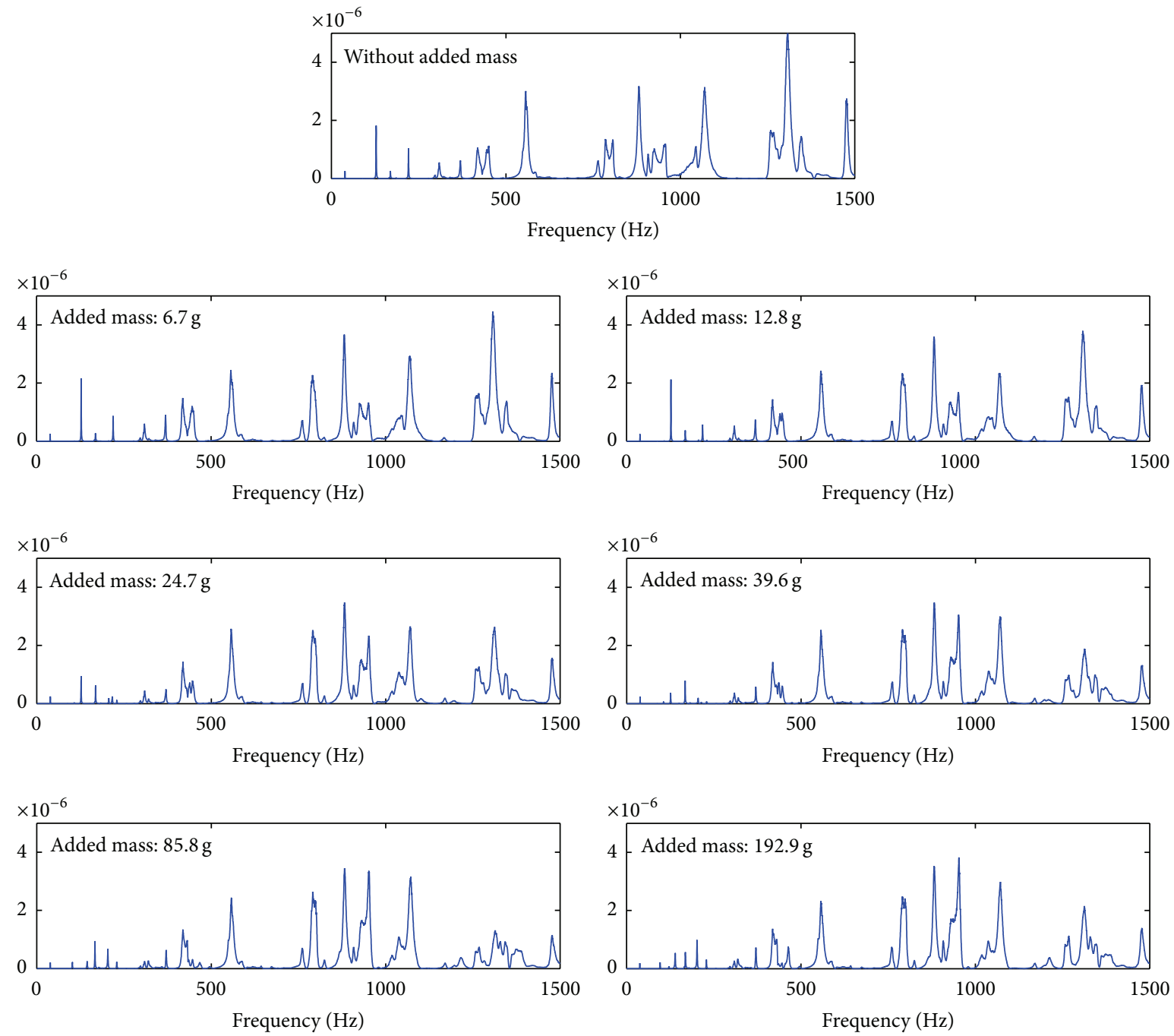

FIGURE 4: Examples of experimental FRFs. The examples correspond to the sensor 1 with the damage in position P1. In the figure, the added mass is indicated. 

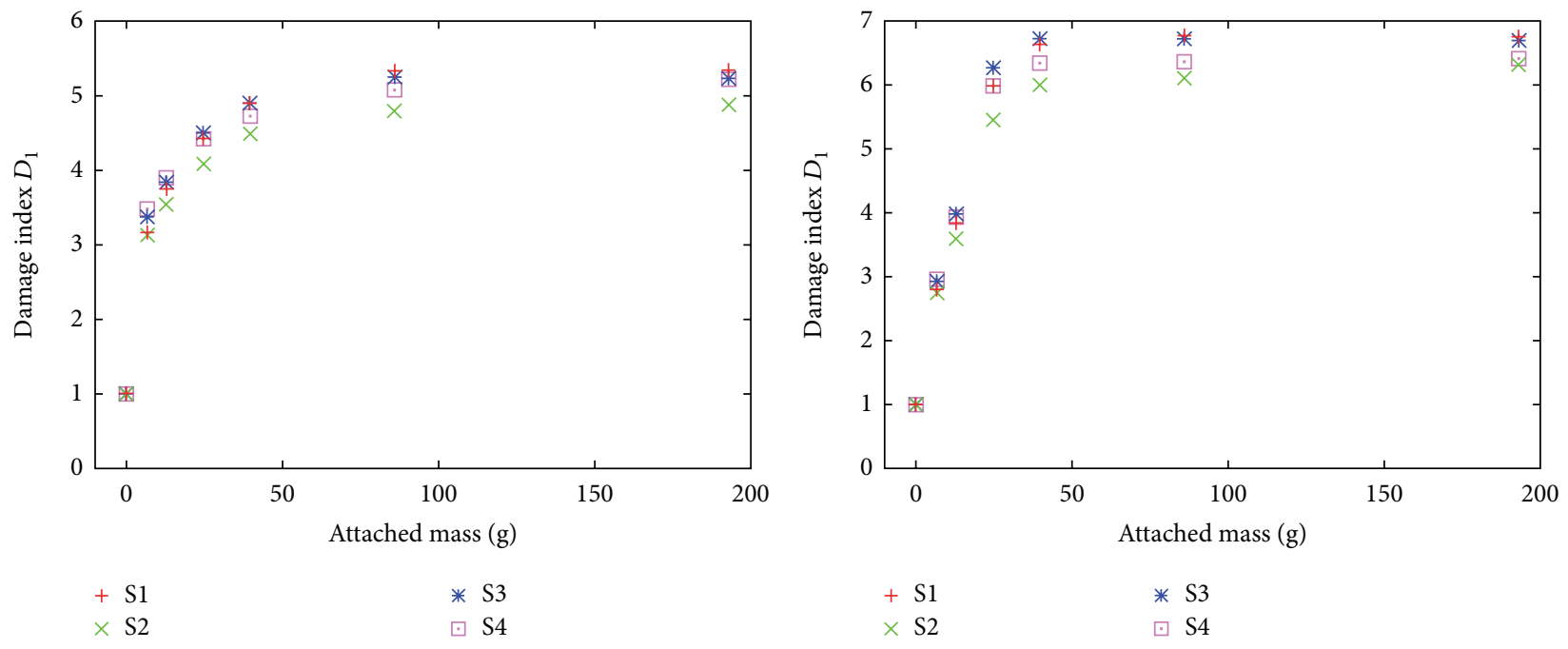

(a)

(b)

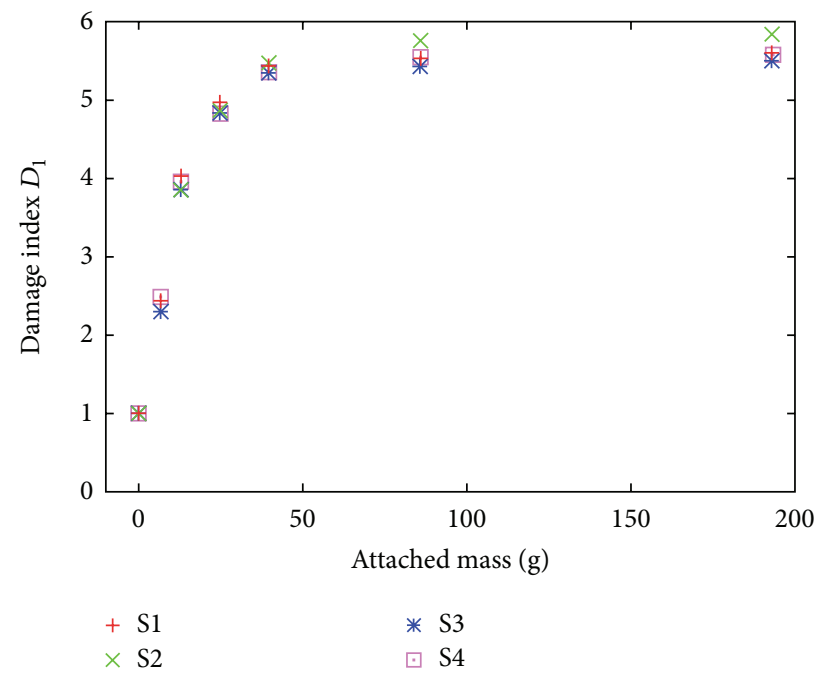

(c)

FIgURE 5: Variation of $D_{1}$ with attached mass. (a) Damage in position P1. (b) Damage in position P2. (c) Damage in position P3.

way, if the energy is distributed over many frequencies, the FRF is very disordered and the SE has a high value. Taking this into account, the SE can be seen as an indicator of the complexity of the vibration, as it will be more complex if more frequencies are involved in the movement.

As an example, in Figure 3 three normalized FRFs can be seen. The first one has only two frequencies of the same amplitude, and its SE is 3.163. The second one has the same frequencies as the previous one but one of them has higher amplitude than the other one, so it is more ordered and its SE has a value of 3.108, which is lower than the first one. The third FRF has three frequencies of equal value and its SE is 3.443. As the energy is distributed over more frequencies, the FRF is more disordered than the first one, so its SE is higher.

The SE can be used as damage index under the assumption that damage changes the amplitude distribution in the
FRF of the structure. That is, if there is only a change in the value of the natural frequencies, but they have the same amplitude as the original ones, then their SE will be the same. The SE will change if the new frequencies have different amplitude than the previous ones.

To obtain the FRF the structure must be excited with an external force (input) and the vibration in a point of the structure must be measured (output). The input energy is distributed between the different natural frequencies depending on physical characteristics of the structure and the position of the actuator and the sensor. The amplitude of each frequency peak of the FRF shows which part of the input energy corresponds to that frequency.

Physically, damage decreases the stiffness of the region of the structure where it is located. It modifies the physical characteristics of the structure and the way that it vibrates, 


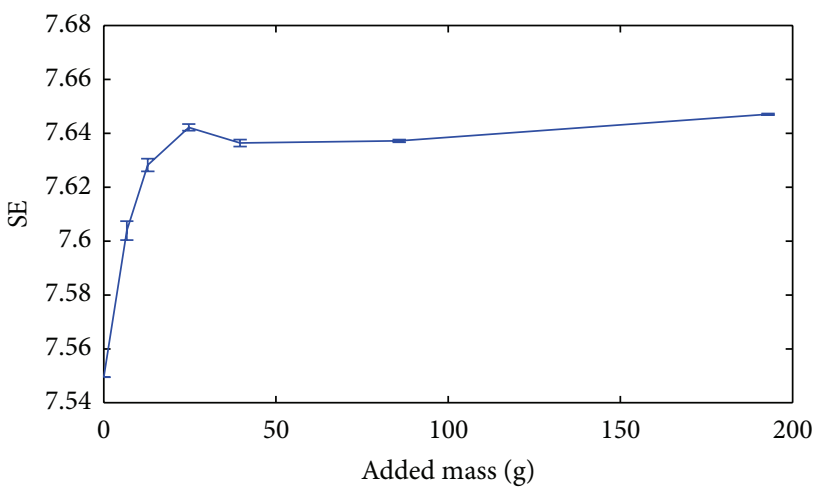

(a)

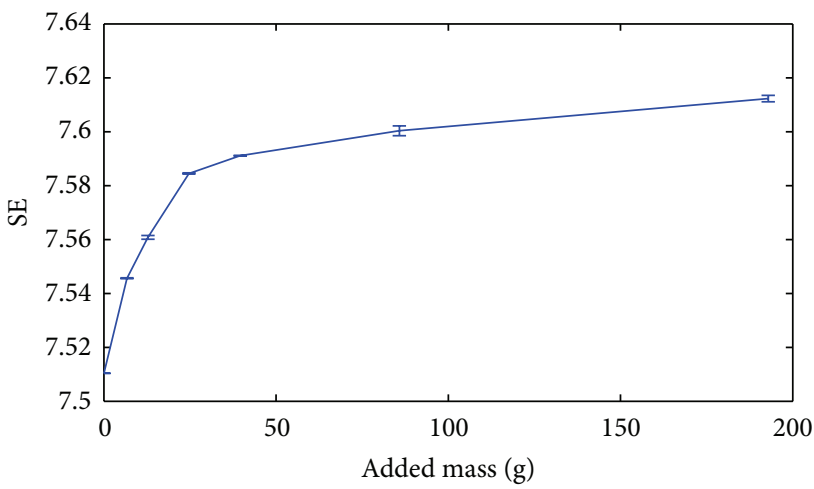

(c)

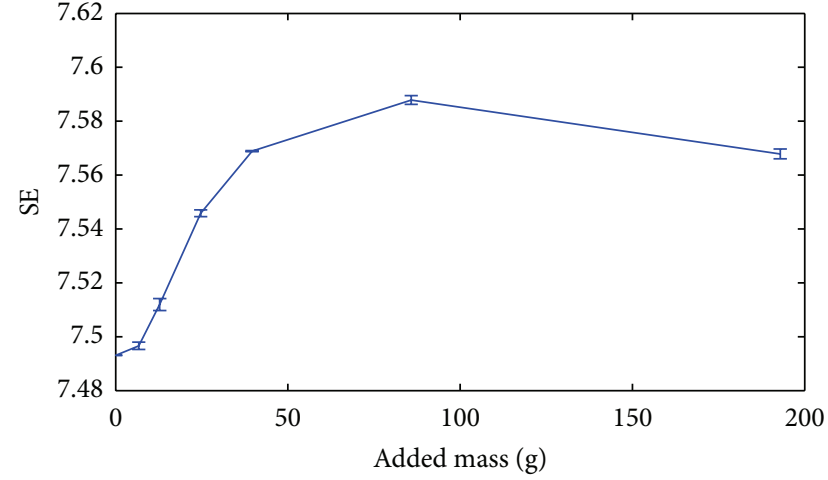

(b)

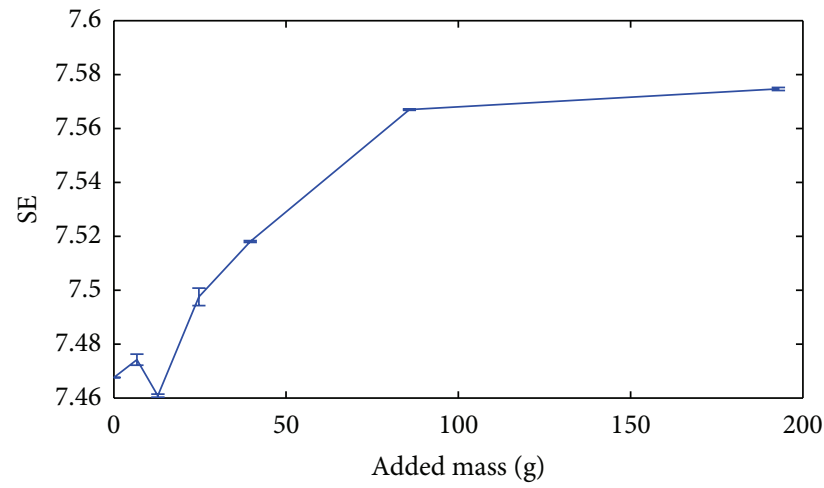

(d)

FIGURE 6: Variation of SE with the added mass in position 1 for: (a) Sensor S1; (b) Sensor S2; (c) Sensor S3; (d) Sensor S4.

TABLE 2: Properties and positions of piezoelectric sensors and actuators.

\begin{tabular}{|c|c|c|c|c|c|c|}
\hline Use & Ceramics & Electrode & Thickness & Diameter & Positions & Notation \\
\hline Actuator & PIC255 & $\mathrm{Ag}$ & $0.5 \mathrm{~mm}$ & $20 \mathrm{~mm}$ & $(42.5,32.5) \mathrm{cm}$ & A1 \\
\hline \multirow{4}{*}{ Sensor } & \multirow{4}{*}{ PIC151 } & \multirow{4}{*}{$\mathrm{Ag}$} & \multirow{4}{*}{$0.5 \mathrm{~mm}$} & \multirow{4}{*}{$10 \mathrm{~mm}$} & $(24.5,21.5) \mathrm{cm}$ & S1 \\
\hline & & & & & $(24.5,43.5) \mathrm{cm}$ & S2 \\
\hline & & & & & $(60.5,21.5) \mathrm{cm}$ & S3 \\
\hline & & & & & $(60.5,43.5) \mathrm{cm}$ & S4 \\
\hline
\end{tabular}

TABLE 3: Added masses and comparison with the total mass of the plate.

\begin{tabular}{lc}
\hline Added masses $(\mathrm{g})$ & Percentage of the mass of the plate \\
\hline 6.7 & 0.14 \\
12.8 & 0.27 \\
24.7 & 0.52 \\
39.6 & 0.83 \\
85.8 & 1.81 \\
192.9 & 4.06 \\
\hline
\end{tabular}

enhancing some frequencies and weakening others. So, the SE of the structure will change in the presence of damage.

Up to the authors' knowledge, the SE has not been used before to identify damage from vibrations in structures. Similar parameters with the same name have been used in other scientific fields, for example, in medicine [4, 5], ecology [6], genomics [7], and acoustics [8], but usually in each different application the SE is defined in a different way. In acoustic emission it has been used with the name of frequency entropy to analyze the obtained signals [9]. A parameter that is similar to SE is the wavelet entropy (WE), which is the Shannon entropy of the wavelet energy obtained from the continuous wavelet transform [10] or of the different frequency bands obtained with the discrete wavelet transform 


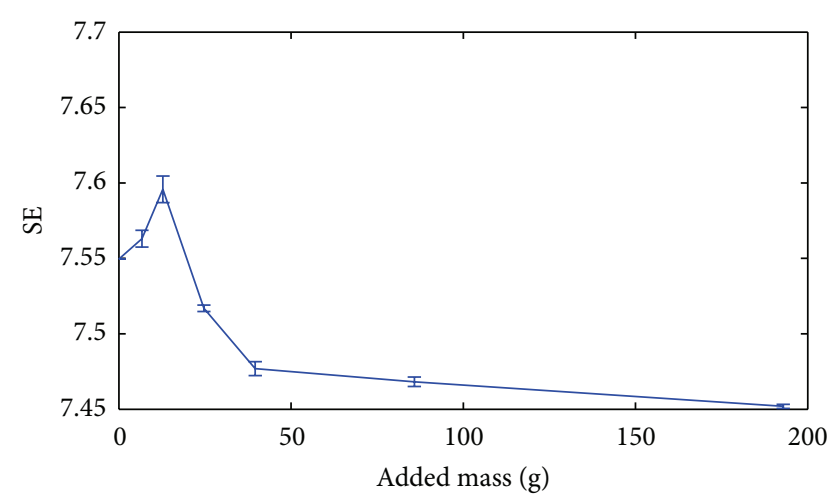

(a)

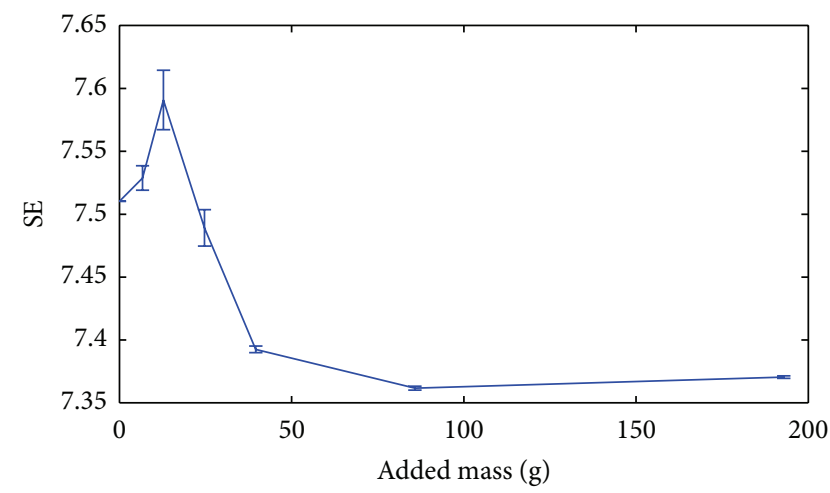

(c)

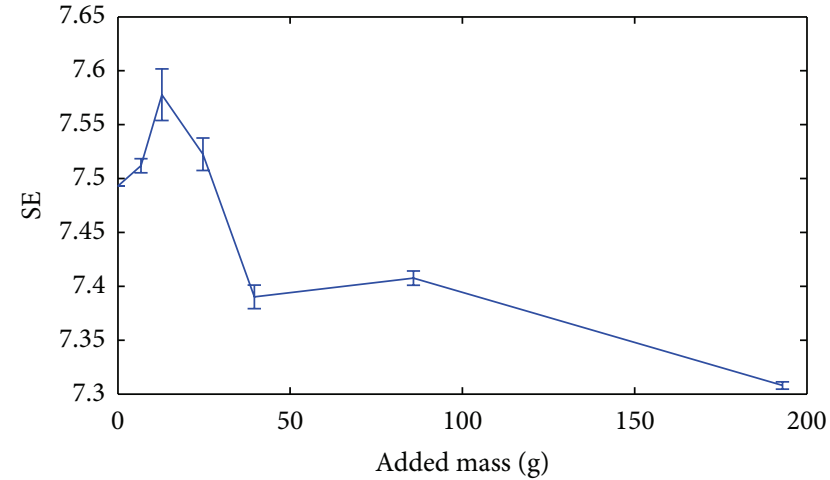

(b)

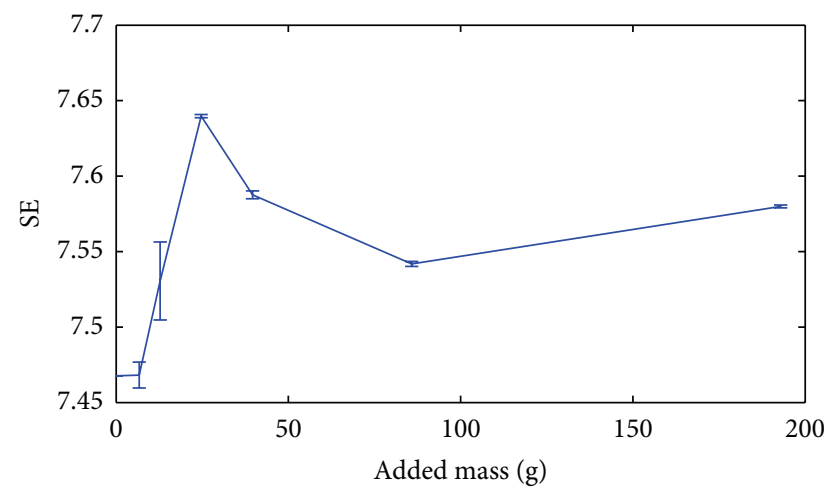

(d)

FIGURE 7: Variation of SE with the added mass in position 2 for (a) Sensor S1; (b) Sensor S2; (c) Sensor S3; (d) Sensor S4.

[11]. The WE have been used successfully to detect damage in structures [12].

\section{Experimental Results}

The experiment was repeated 15 times in the undamaged case and 5 times in each damaged plate to minimized ambient and random errors. The sample frequency was $4096 \mathrm{~Hz}$ and the frequency content of the excitation signal was up to $1500 \mathrm{~Hz}$. The power spectral densities (PSD) of the excitation and the output were computed using Matlab and the Welch algorithm. The PSD of the output were divided by the PSD of the excitation to obtain the FRF. Some examples of FRFs of the plate with different added mass can be seen in Figure 4.

The change of these experimental FRFs with damage has been studied in previous works $[2,3]$. This change was quantified using the following parameter:

$$
D_{1}=\sum_{f_{i}=f_{\min }}^{f_{i}=f_{\max }} \sqrt{\left|\operatorname{FRF}_{\text {undamaged }}\left(f_{i}\right)-\mathrm{FRF}_{\text {damaged }}\left(f_{i}\right)\right|}
$$

The difference between the FRF of the intact and damaged plate increases with damage, so $D_{1}$ can be used as damage index. In Figure 5 it is shown the variation of $D_{1}$ with the added mass for each damage location. It can be seen that the change in the FRF increases very fast with low damage, but for massed higher than $39.6 \mathrm{~g}, D_{1}$ remains almost constant in all cases.

To study the performance of the SE as a damage index, it was computed for each experiment, and for each undamaged and damaged plate the mean and standard deviation were calculated. The variation of the SE with the added mass for each position of the damage is shown in Figures 6, 7, and 8, where error bars are added to show the standard deviation due to the different experiments.

As can be seen in Figures 6-8, there is a trend of the SE with the added masses. With damage in positions 1 and 3 , the tendency is that the SE rises with mass, but not in monotonic form. On the other side, with damage in position 2 , the tendency is that the SE decreases with mass, except in the case of sensor 4, which oscillates instead of having a trend. This means that damage in positions 1 and 3 produces more relevant frequencies in the FRF; that is, the energy distributes between more frequencies in the FRF. In the opposite side, with damage in position 2 there are less relevant frequencies. Using SE as a measure of the complexity, damage in positions 1 and 3 makes the vibration more complex, while damage in position 2 decrease its complexity.

This different behaviour of the SE with damage location can be explained by taking into account that the presence of a mass in a point of the plate makes its movement more 


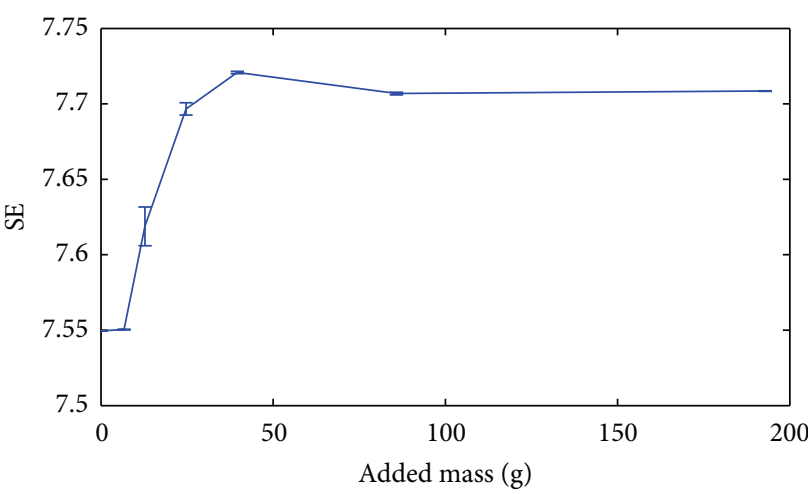

(a)

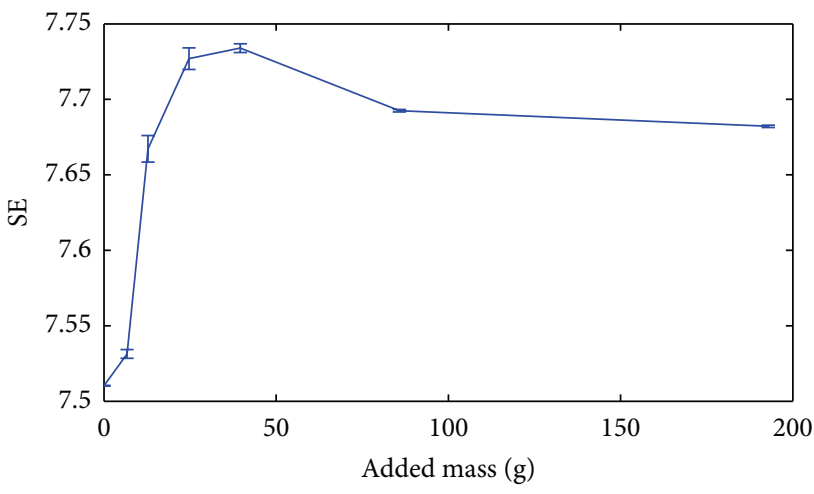

(c)

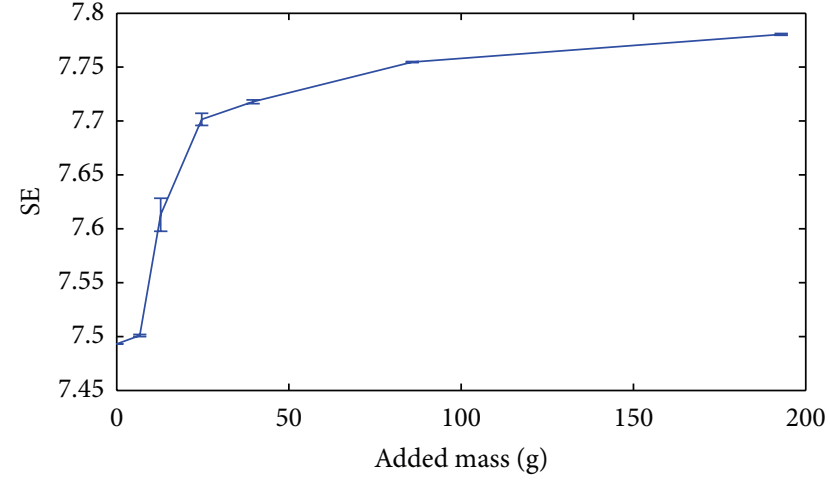

(b)

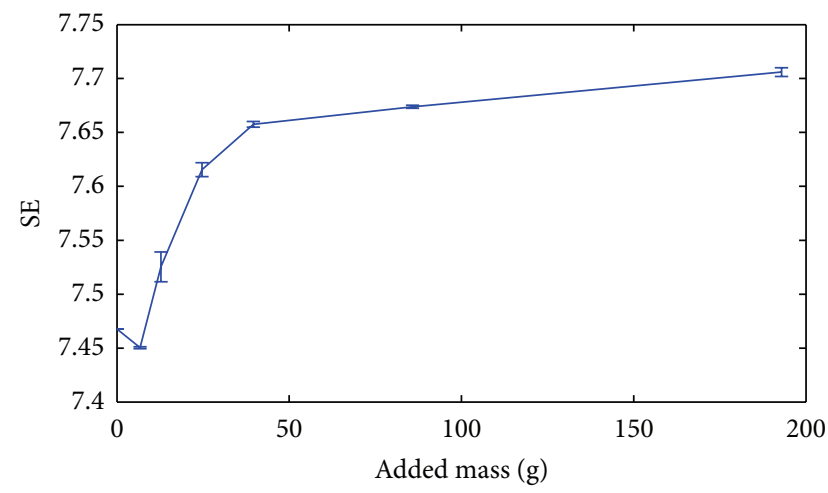

(d)

FIgURE 8: Variation of SE with the added mass in position 3 for (a) Sensor S1; (b) Sensor S2; (c) Sensor S3; (d) Sensor S4.

difficult. So the normal modes with an antinode which is near the damage location will be disturbed, and their influence in the vibration will decrease. In positions 1 and 3 the disturbed modes must be the principal ones, so the influence of less important modes is enhanced and the complexity of the vibration is higher. In the case of position 2, the disturbed modes have probably low relevance in the vibration and more energy goes to the principal ones, making the movement less complex. The damage index $D_{1}$ has the same behaviour in the three damage locations because it measures the difference between the FRFs of the undamaged and damaged plates, so this difference always increases with added mass.

For low added mass the SE changes very quickly, but for masses around $39.6 \mathrm{~g}$ and $85.8 \mathrm{~g}$ it becomes almost constant. It seems that when we have a high value of mass, a further increase does not change the amplitude distribution in the FRF and the SE remains constant. The same behaviour was found for damage index $D_{1}$, which is a direct measure of the change in the FRF. So it seems that the FRF changes very little when a certain value of damage is reached. The reason for this saturation of the damage indexes can be that, as have been said before, an increase of the mass in a point of the plate decreases its movement. For a low value of the mass, the point can still move. But for a high value, there is a strong limitation of the movement and it remains still. As it cannot move, a further increase of the mass will not have any effect on it.
It is possible that the results can be influenced by the fact that the masses were not fixed to the plate, but it seems that it is not the case. The possible jump of the mass on the plate could introduce a nonlinear effect that increases with weight. In the experimental results, for low masses the SE follows a trend and for high masses it has an approximately constant value. If the effect of the jumps had been important, the SE would have increased with high masses as the FRF would have been more complex.

\section{Conclusions}

The importance of composite structures in aerospace industry is increasing in the last years, making the development of damage identification methods adapted to this kind of materials necessary. A new damage detection technique has been experimentally tested on a CFRP plate. It is based on the use of the spectral entropy (SE) of the vibrations of the CRFP structure as damage index. This parameter measures the energy distribution between the resonant frequencies of the plate and is related with the complexity of the vibration. It has been proved that the SE changes with damage, and this change is higher than the variations in SE due to different measures of the FRF. Furthermore, the variation in the SE with damage follows a trend: the SE increases or decreases 
(depending of the location) as damage increases. It means that the SE can be related with the quantity of damage in a given position and with more research, with its localization. In our experimental tests it was found that the variation of SE with damage is not always mononotic, which can make it difficult for it to be used in a real situation. It can be due to our experimental arrangement, or damage implementation, or to other reasons. To clarify this point and improve our understanding of the theoretical behaviour of the SE, a numerical investigation must be carried out.

The advantage of the SE over other damage indexes based on FRF is that a change in the ambient or external conditions of the structure (e.g., the temperature) can produce a change in the amplitude of the FRF or a translation of it, so many false positives can appear. The SE is insensitive to these changes, which makes it a very promising damage index for SHM, although more theoretical and experimental research is necessary.

\section{Conflict of Interests}

The authors declare that there is no conflict of interests regarding the publication of this paper.

\section{References}

[1] Y. J. Yan, L. Cheng, Z. Y. Wu, and L. H. Yam, "Development in vibration-based structural damage detection technique," Mechanical Systems and Signal Processing, vol. 21, no. 5, pp. 2198-2211, 2007.

[2] P. Moreno-García, Simulations and experimental tests of vibrations on carbon fiber composite material plates and damage detection with frequency response function and the wavelet transform [Ph.D. thesis], University of Granada, 2012, (Spanish), http://hera.ugr.es/tesisugr/21009417.pdf .

[3] P. Moreno-García, E. Castro, L. Romo-Melo, A. Gallego, and A. Roldán, "Vibration tests in CFRP plates for damage detection via non-parametric signal analysis," Shock and Vibration, vol. 19, no. 5, pp. 857-865, 2012.

[4] M. Jiahai, W. Xueyan, X. Yonggang et al., "Spectral entropy monitoring reduces anesthetic dosage for patients undergoing off-pump coronary artery bypass graft surgery," Journal of Cardiothoracic and Vascular Anesthesia, vol. 26, no. 5, pp. 818821, 2012.

[5] J. Morgaz, M. D. M. Granados, J. M. Domínguez et al., "Evaluation of spectral entropy to measure anaesthetic depth and antinociception in sevoflurane-anaesthetised Beagle dogs," Veterinary Journal, vol. 188, no. 3, pp. 352-355, 2011.

[6] N. Zaccarelli, B.-L. Li, I. Petrosillo, and G. Zurlini, "Order and disorder in ecological time-series: introducing normalized spectral entropy," Ecological Indicators, vol. 28, pp. 22-30, 2013.

[7] V. R. Chechetkin and V. V. Lobzin, "Spectral entropy criteria for structural segmentation in genomic DNA sequences," Physics Letters A: General, Atomic and Solid State Physics, vol. 328, no. 1, pp. 79-86, 2004.

[8] N. C. Han, S. V. Muniandy, and J. Dayou, "Acoustic classification of Australian anurans based on hybrid spectral-entropy approach," Applied Acoustics, vol. 72, no. 9, pp. 639-645, 2011.

[9] R. Unnthorsson, T. P. Runarsson, and M. T. Jonsson, "AE entropy for the condition monitoring of CFRP subjected to cyclic fatigue," Journal of Acoustic Emission, no. 26, pp. 229-239, 2008.

[10] R. Piotrkowski, E. Castro, and A. Gallego, "Wavelet power, entropy and bispectrum applied to $\mathrm{AE}$ signals for damage identification and evaluation of corroded galvanized steel," Mechanical Systems and Signal Processing, vol. 23, no. 2, pp. 432445, 2009.

[11] D. M. Tavares and L. S. Lucena, "Entropy analysis of stochastic processes at finite resolution," Physica A: Statistical Mechanics and Its Applications, vol. 357, no. 1, pp. 71-78, 2005.

[12] W.-X. Ren and Z.-S. Sun, "Structural damage identification by using wavelet entropy," Engineering Structures, vol. 30, no. 10, pp. 2840-2849, 2008. 

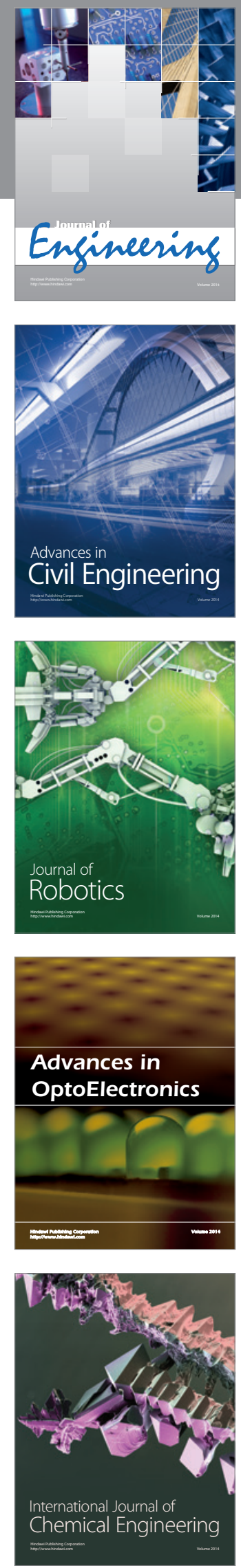

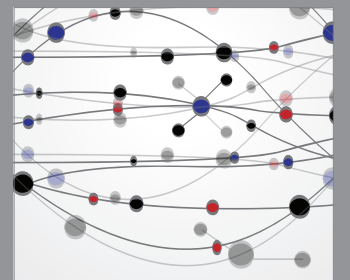

The Scientific World Journal
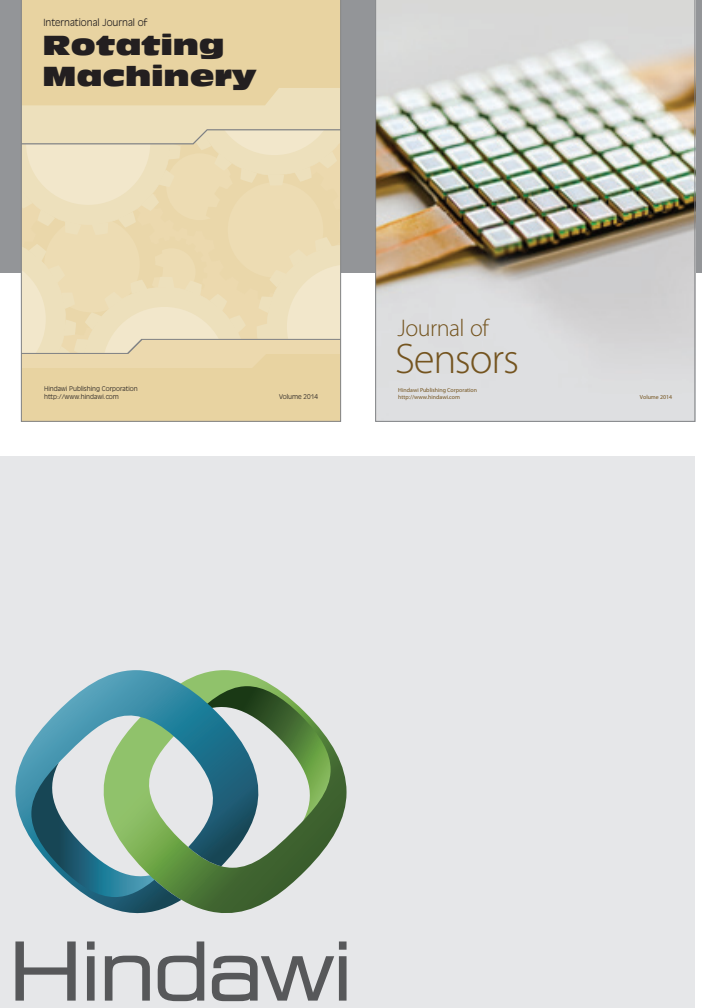

Submit your manuscripts at http://www.hindawi.com
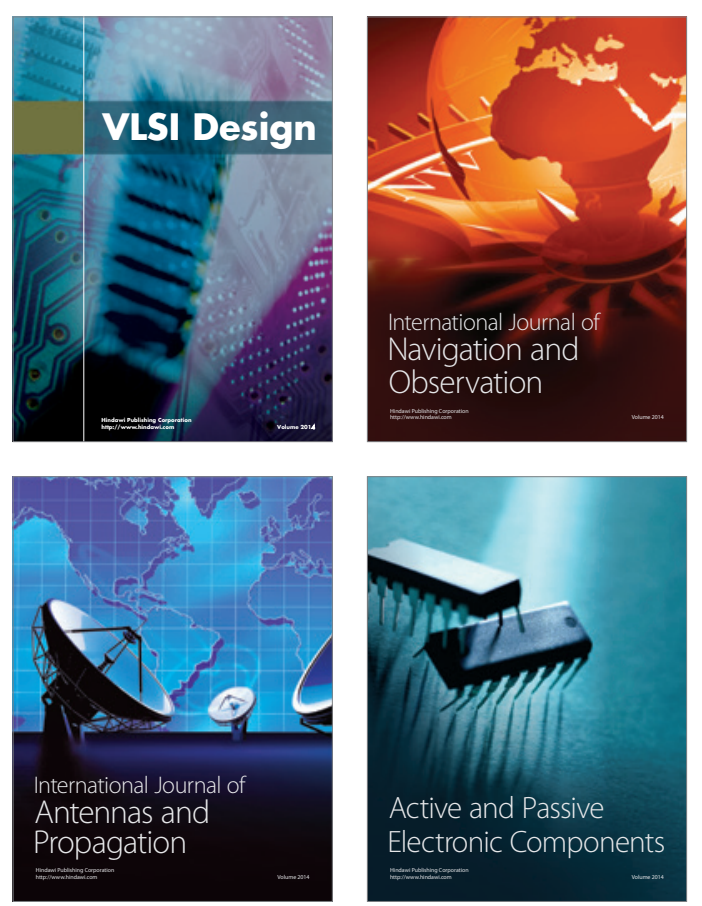
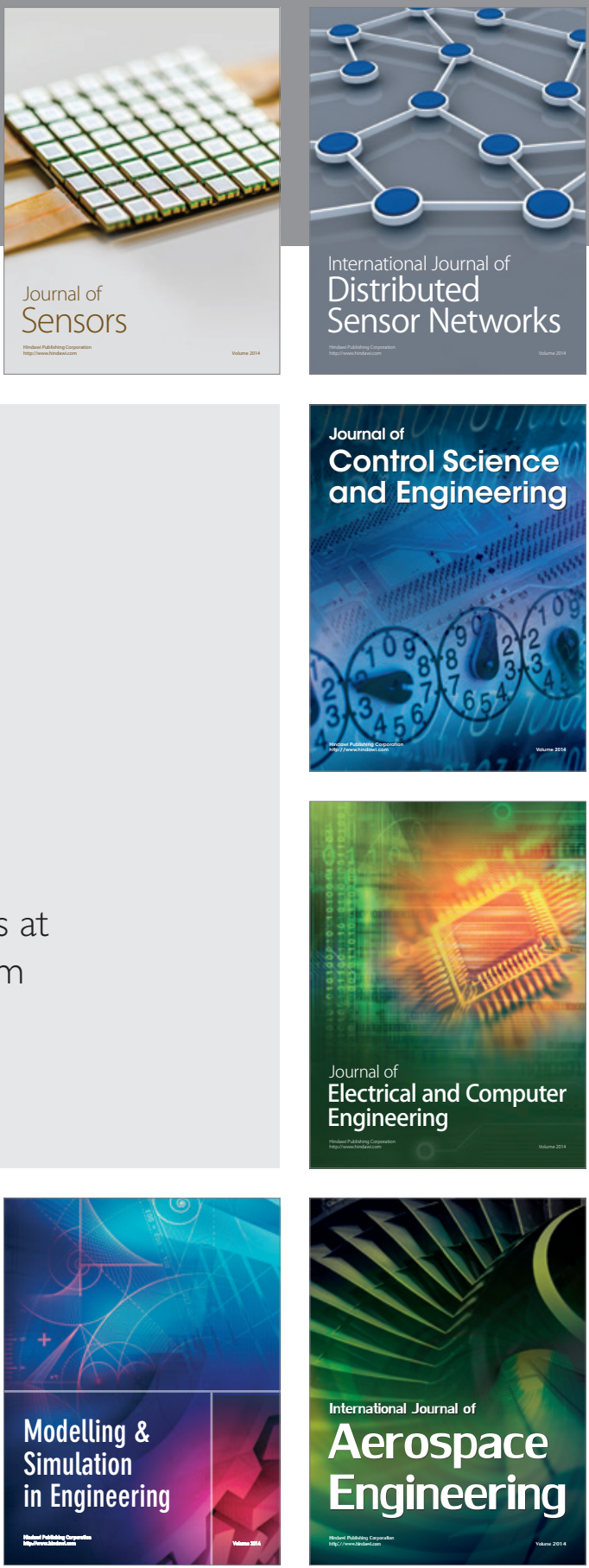

Journal of

Control Science

and Engineering
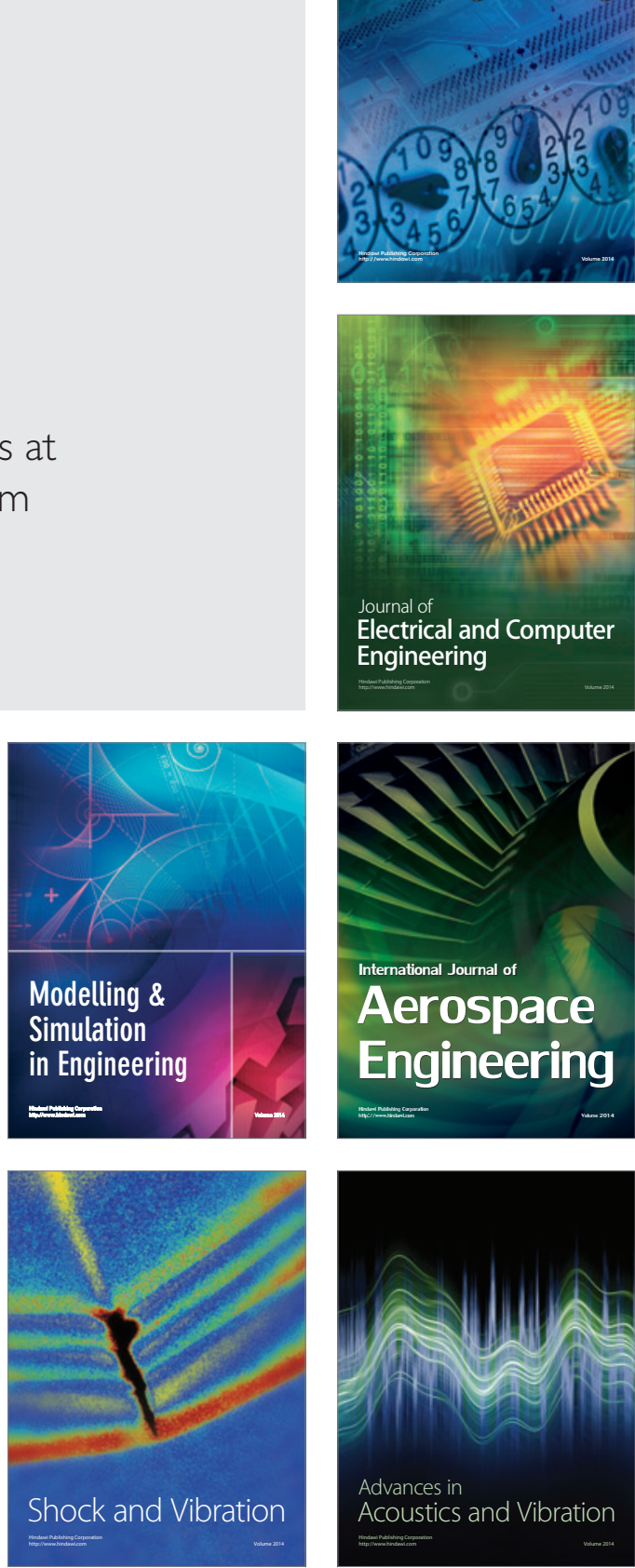